\title{
La seguridad con perspectiva de género. Experiencias en Cataluña
}

\section{La seguridad con perspectiva de género. Experiencias en Cataluña}

Ana Belen Almecija Casanova*

Recibido: 04-11-2021

Aceptado: 28-12-2021

\section{Resumen:}

La seguridad es un derecho fundamental y una condición indispensable para el libre ejercicio de la libertad. Tradicionalmente se ha considerado un bien objetivo gestionado a través de políticas neutras respecto del género, que han dejado de lado las demandas y las necesidades específicas de las mujeres, tanto de las ciudadanas como de las profesionales de la seguridad. Este artículo tiene como objetivo abordar algunos ámbitos desde los que se puede trabajar la perspectiva de género en la seguridad - desde una perspectiva transversal e interseccional - y también explicar algunas

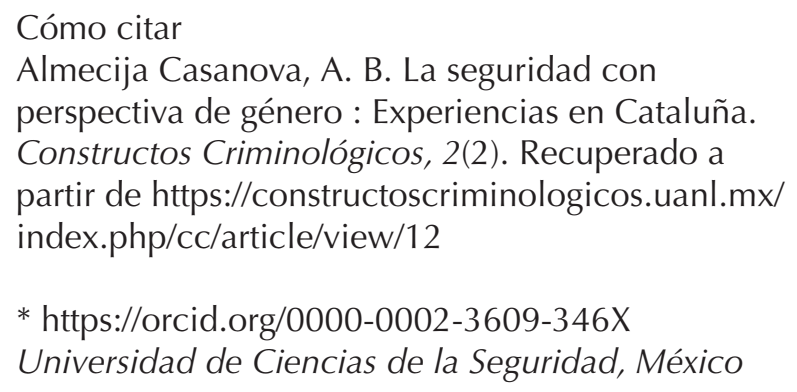
perspectiva de género : Experiencias en Cataluña. Constructos Criminológicos, 2(2). Recuperado a partir de https://constructoscriminologicos.uanl.mx/ index.php/cc/article/view/12

* https://orcid.org/0000-0002-3609-346X Universidad de Ciencias de la Seguridad, México

experiencias concretas al respecto que se han desarrollado en Cataluña a nivel normativo y de protocolos de actuación.

Palabras Clave: Seguridad, género, perspectiva de género,

\section{Abstract}

Security is a fundamental right and an essential condition for free exercise of freedom. Traditionally, gender-neutral policies have been considered a good management of it. However, that has ignored the specific demands and needs of women, both female civilians and security professionals. The aim of this article is to address some areas in which gender perspective in security can be explored - from a transversal and intersectional perspective- and to share some specific tools (regulations and operational protocols) that have been developed in Catalonia.

Keywords: Security, gender, gender perspective 


\section{LA PERSPECTIVA DE GÉNERO COMO ESTRATEGIA PARA ALCANZAR LA IGUALDAD}

Este artículo tiene como objetivo concretar algunos ámbitos desde los que se puede trabajar la seguridad con perspectiva de género ${ }^{1}$, así como fundamentar la necesidad de hacerlo - e incluso la obligatoriedad, ya que así lo establece la normativa - desde un enfoque transversal, multidisciplinar e interseccional.

La seguridad es un derecho fundamental y una condición indispensable para el libre ejercicio de la libertad ${ }^{2}$. Tradicionalmente se ha considerado un bien objetivo gestionado a través de políticas neutras respecto del género, que han dejado de lado las demandas y las necesidades específicas de las mujeres, tanto como ciudadanas como de las profesionales y expertas en seguridad.

1 Entendido en un sentido amplio, incluyo también que el enfoque de género nos puede llevar a plantear otra manera de hacer las cosas. Por ejemplo, la gestión alternativa de conflictos aplicada al ámbito de la seguridad, que permite una mejor garantía de las funciones otorgadas a los cuerpos de seguridad al servicio de los derechos de la ciudadanía y el fortalecimiento del modelo de seguridad preventivo (Ayguasenosa, 2009).

2 En España hay un reparto competencial entre Estado y Comunidades Autónomas, el artículo 149.1.29 de la Constitución indica que es competencia exclusiva del Estado la "seguridad pública, sin perjuicio de la posibilidad de creación de policías por las Comunidades Autónomas en la forma que se establezca en los respectivos Estatutos en el marco de lo que disponga una ley orgánica" El artículo 164 del Estatuto de Autonomía de Cataluña establece las funciones de la Generalitat en seguridad pública y el artículo 163 en seguridad privada. En este artículo se hará referencia a normativa estatal y a normativa autonómica de Cataluña.
Si la seguridad es el instrumento que debe garantizar el vivir y el convivir con tranquilidad a las personas, es necesario incorporar la perspectiva de género tanto en la misma definición de seguridad como en todas las acciones que plantea partiendo de la constatación que a las mujeres y a los hombres se les asignan roles sociales diferentes y que no existe el ciudadano medio "neutro" en el género. A partir del análisis de necesidades de la población en materia de seguridad y de la definición de las políticas públicas de seguridad con perspectiva de género es cómo y cuándo podremos hablar de actuaciones dirigidas verdaderamente a toda la población (García, 2009).

La incorporación de la perspectiva de género integra la igualdad de género en las organizaciones públicas y privadas de un país en políticas centrales o locales, y en programas de servicios y sectoriales. ${ }^{3}$ Supone tomar en consideración las diferencias entre mujeres y hombres en un ámbito o actividad para el análisis, planificación, diseño y ejecución de políticas, teniendo en cuenta el modo en que las diversas actuaciones, situaciones y necesidades afectan a las mujeres. Este enfoque permite visualizar mujeres y hombres en su dimensión biológica, psicológica, histórica, social y cultural, y también permite encontrar líneas de reflexión y actuación para la erradicación de las desigualdades. ${ }^{4}$

3 Vid. ONU mujeres https://www.unwomen.org/es/ how-we-work/un-system-coordination/gender-mainstreaming 4 Así la define la Ley catalana 17/2015, de 21 de julio, de igualdad efectiva de mujeres y hombres. https://www.boe. es/diario_boe/txt.php?id=BOE-A-2015-9676 
La Plataforma para la Acción de la Cuarta Conferencia Mundial sobre Mujeres de Naciones Unidas que se celebró en Pekín en $1995^{5}$, asumió de forma explícita la transversalidad de género ("mainstreaming") e hizo un requerimiento a los gobiernos y otros actores a promocionar una política activa $y$ visible del mainstreaming de género, en todas las políticas y programas, con el objetivo de que antes de que se tomen las decisiones, se realice un análisis de los efectos producidos en mujeres y hombres, respectivamente.

Las conclusiones convenidas de la ECOSOC de $1997^{6}$ definían la incorporación de una perspectiva de género como: "El proceso de evaluación de las consecuencias para las mujeres y los hombres de cualquier actividad planificada, inclusivamente las leyes, políticas o programas, en todos los sectores y a todos los niveles. Es una estrategia destinada a hacer que las preocupaciones y experiencias de las mujeres, así como de los hombres, sean un elemento integrante de la elaboración, la aplicación, la supervisión y la evaluación de las políticas y los programas en todas las esferas políticas, económicas y sociales, a fin de que las mujeres y los hombres se beneficien por igual y se impida que se perpetúe la desigualdad. El objetivo final es conseguir la igualdad [sustantiva] entre los géneros"

$5 \quad$ Vid. Declaración y Plataforma de Acción de Beijing https://beijing20.unwomen.org/ /media/headquarters/attachments/sections/csw/bpa_s_final_web.pdf

6 Vid. Informe del Consejo Económico y Social correspondiente a 1997 https://undocs.org/pdf?symbol=es/A/52/3/REV.1(SUPP)
Por lo tanto, la igualdad es el objetivo de desarrollo general y a largo plazo, mientras que la incorporación de una perspectiva de género es un conjunto de enfoques específicos y estratégicos, así como procesos técnicos e institucionales que se adoptan para conseguir este objetivo.

La igualdad es, así mismo, un principio fundamental en la Unión Europea donde se integró la perspectiva de género como un planteamiento político oficial para conseguir la igualdad "incorporar la perspectiva de género puede marcar la diferencia entre conseguir dar respuesta a las necesidades de la población o fracasar en el intento, y entre una buena política o una política ineficaz, o incluso contraproducente" (Shreeves, 2019)

\section{TRABAJAR LA SEGURIDAD CON PERSPECTIVA DE GÉNERO}

Las políticas de seguridad deben visibilizar las necesidades y demandas específicas de las mujeres en un sentido amplio como ciudadanas y también de las profesionales de la seguridad. La perspectiva de género no es una "revisión" que debe hacerse a posteriori de un documento, de una acción o de una política pública, debe existir desde el momento inicial en las metodologías de investigación, diagnóstico, planificación e implantación de medidas y también en la evaluación de las mismas.

La formación del personal de la policía y de la seguridad privada es esencial, y debe contener temas sobre igualdad, a nivel de sensibilización y un enfoque teórico, pero también práctico y operativo. Además, se debe incidir en capacitar 
sobre aquellos delitos e infracciones en los que hay un mayor impacto de género como son las relacionadas con las violencias machistas ${ }^{7}$, también en cómo abordar algunas conductas que puede que no estén sancionadas pero no por ello quien la sufre debe quedar desamparo ${ }^{8}$.

El trabajo por hacer es extenso: sensibilizar, conocer la normativa, los protocolos de actuación y tener previstas todas las situaciones posibles en las que se debe proteger a la mujer y planificar todas las medidas posibles para garantizar su integridad física y psíquica. A la vez, incidir en un discurso que interpele directamente a los agresores ${ }^{9}$.

No se puede obviar que es un terreno en el que todavía hay resistencias y obstáculos ${ }^{10}$ en

$7 \quad$ En España en 2020 fueron 45 las víctimas de asesinato $\mathrm{u}$ homicidio en manos de su pareja o expareja. Puede consultarse el dato en el Anuario Estadístico del Ministerio del Interior, así como del sistema de seguimiento integral en los casos de violencia de género (sistema viogén) http://www. interior.gob.es/documents/642317/1204854/Anuario+Estad\%C3\%ADstico+del+Ministerio+del+Interior+2020/94d9cf1b-889f-4119-b4c4-09926630a29e

8 Algunos tipos de acoso sexual - como el acoso verbal callejero- no están sancionados de la misma forma en todos los países y en muchos ni se contempla, pero pueden causar malestar en quien lo recibe y provocarle una fuerte sensación de inseguridad.

9 En octubre de 2021 la Policía de Escocia lanzó la campaña "That guy" https://that-guy.co.uk/ con el objetivo de que los hombres asuman la responsabilidad de poner fin a la violencia sexual cambiando sus actitudes y comportamientos hacia las mujeres y desafiando los de sus pares.

10 María Naredo y Praxágora Cooperativa dedican un apartado concreto a estos obstáculos y cómo superarlos en la relación a, por ejemplo:

- Discursos de miembros de la organización que niegan la discriminación de la mujer, la invisibilización de sus necesidades e incluso la violencia que se ejerce contra ella.

- No comprender la necesidad de incorporar la perspectiva de género por creer que la seguridad es neutra y "no tiene género".

- No entender que una medida que es útil para garantizar la seguridad de mujeres puede serlo también para los hombres y eso no significa que no tenga perspectiva de género (se puede llegar a la misma conclusión que sin ella, pero al haberse investigado, planificado e implantando con perspectiva de género nos garantiza que las mujeres han podido dar su opinión - como ciudadanas y como expertas y profesionales- y participar del proceso).

- Considerar que no se precisa un perfil técnico o especialización en género para implantar medidas, dejando esta tarea a un miembro de la organización que se considere con sensibilidad sobre el tema, pero sin formación alguna en igualdad.

- Creerque es innecesario trabajar la seguridad con perspectiva de género ya que la organización o la entidad es suficientemente paritaria, no constan denuncias de acoso sexual ni de discriminación por razón de sexo, no constan demandas específicas de mujeres, etc.

- Falta de tiempo, recursos, desconocimiento de cómo llevar a cabo estos procesos.

Guía para la elaboración de diagnósticos de seguridad con visión de género en el ámbito rural y urbano (véase la referencia en Trabajos citados). 
Para superar estos obstáculos se precisa como punto de partida la capacitación en igualdad de todas las personas de la organización donde además de conceptos y normativa, se compartan materiales, resultados de investigaciones que muestren las diferentes necesidades de seguridad y de la percepción de seguridad de hombres y mujeres, experiencias en otras organizaciones y cómo se traslada todo ello de manera operativa a las actuaciones.

En los siguientes apartados explicaré brevemente solo algunos de los puntos que se pueden explorar para trabajar la seguridad con perspectiva de género y dedicaré unos apartados concretos a la experiencia de Cataluña en este ámbito.

\section{LAS METODOLOGÍAS DE INVESTIGACIÓN}

No es objeto de este artículo explicar las diferentes metodologías de investigación, pero sí señalar algunas que han sido de gran utilidad para trabajar la seguridad con perspectiva de género.

Las metodologías cuantitativas son imprescindibles peroinsuficientes, porloquedebencomplementarse siempre con metodologías cualitativas entre las cuales se ha de contar especialmente con aquellas que sean participativas. Unas y otras han de ser diseñadas con perspectiva de género.

Igareda, N., \& Bodelón, E. (2014) defienden que la opción del trabajo cualitativo (Vallés, 1997, 2002; Quivy y Van Campenhoudt, 1992) permite obtener información valiosa que no es posible obtener a través de métodos únicamente cuantitativos. Igualmente permite el análisis de nuevos conceptos, que la investigación quizás no había planteado en las hipótesis iniciales. Muchas investigadoras feministas han preferido la investigación cualitativa pues permite a las víctimas de la violencia sexual articular experiencias con sus propias palabras (Campbell et al. 2009; Jordan, 2001, 2004, 2008; Kelly, 2008; Stanko, 1990)

En cuanto a las cualitativas destaco una herramienta metodológica de gran interés como son las marchas exploratorias que se originaron a principio de los años noventa en las ciudades de Montreal y Toronto. Las marchas exploratorias son una herramienta que permite analizar desde una perspectiva feminista interseccional cómo la configuración física, social y simbólica de los espacios urbanos condiciona la percepción de (in)seguridad (Observatorio Noctámbul@s, 2018 $)^{11}$. Consisten en la evaluación realizada en terreno que hace un grupo de mujeres de un espacio, ya puede ser de un barrio de un municipio o de una zona concreta que se considera especialmente problemática. Se realizan recorriendo en grupo la zona y se evalúa a través de una lista de chequeo, a la vez que se anotan las vivencias, sensaciones $y$ experiencias que manifiestan las participantes, se anotan los aspectos que generan inseguridad pero también los que proporcionan autonomía y libertad. El objetivo es hacer una propuesta de soluciones viables de mejoramiento del diseño urbano que disminuyan la percepción de inseguridad de las personas.

11 Se puede ver un ejemplo de experiencias de marchas exploratorias en el Informe anual 2017-2018 del Observatorio Noctámbul@s donde además se transcriben las manifestaciones de las participantes. 
También hay experiencias muy interesantes como la del municipio de Bilbao, que ha creado una herramienta participativa conocida como "Mapa de los puntos críticos"12, un proyecto del ayuntamiento con participación ciudadana, con la finalidad de mejorar los puntos de la ciudad que no tienen visibilidad, accesibilidad o son aislados y por lo tanto producen inseguridad, sobre todo en las mujeres. Cualquier persona puede entrar a la web para consultar el mapa y también enviar una propuesta argumentada para que se añada en él un punto crítico.

Este proyecto es continuación de otro anterior, el "Mapa de la Ciudad Prohibida en los Distritos de Bilbao"13, iniciativa que pretendía aumentar la sensación de libertad y seguridad de las mujeres en su entorno cercano, visibilizando las experiencias de las mujeres y sus necesidades en el uso de los espacios públicos, identificando los de baja calidad y haciendo recomendaciones, tomando como punto de partida las aportaciones que habían hecho las vecinas.

\section{LAS DIFERENTES PERCEPCIONES DE LA (IN) SEGURIDAD}

Las mujeres y los hombres se relacionan con el entorno y con las personas de manera diferenciada, lo cual provoca un impacto en la

12 Vid. https://www.donostia.eus/info/ciudadano/igualdad_plan.nsf/voWebContenidosld/NT000009AE?OpenDocument\&idioma $=$ cas\&id $=$ A374066376363\&cat $=\& d o c=D$

13 Puede descargarse en https://www.bilbao.eus/cs/ Satellite $? c=$ Page $\&$ cid $=3000082641 \&$ language $=$ es $\&$ pagei $\mathrm{d}=3000082641$ \&pagename $=$ Bilbaonet\%2FPage\%2FBIO_contenidoFinal percepción de la seguridad. Hay que tener en cuenta que las mujeres de manera continuada sufren una serie de conductas que no siempre están tipificadas al Código penal como delito. Estas "ofensas cotidianas" a menudo son difícilmente denunciables y a pesar de que la normativa administrativa sí permitiría sancionar algunos comportamientos, otros muchos quedan fuera de lo que han previsto las leyes, por lo tanto, su detección y su abordaje es difícil, especialmente si se busca solo una respuesta punitiva en vez de tratarlo desde la sensibilización, el civismo y el respeto.

Las experiencias vividas personalmente, las vivencias compartidas por amigas, compañeras o familiares o hechos conocidos a través de la prensa o redes sociales, pueden poner a algunas mujeres en alerta o hacerlas sentir miedo ante situaciones que quizás para otra persona no serían consideradas peligrosas o motivo de incomodidad o preocupación. Como recuerda Ortiz (2014) citando a Michaud (2005), si en una calle donde el $100 \%$ de las personas que viven son mujeres, a una la violan, las consecuencias no recaen solo sobre el $1 \%$, esa mujer a la que han violado, sino sobre el 100\% de las mujeres, ya que ese hecho aumentará la percepción de inseguridad y el miedo de las mujeres que viven allí, porque sienten que les puede pasar a ellas también.

Las mujeres tienen un índice de percepción de la inseguridad tres veces superior al de los hombres, aunque el riesgo registrado de sufrir actos violentos contra la persona, especialmente en lo que se refiere a las agresiones, es inferior al de los hombres. Además, no existe una correlación entre el riesgo de victimización 
hecho público por actos delictivos violentos y el miedo que tienen de ser víctimas de estos actos. Los hombres jóvenes, que son los que dicen que se sienten más seguros, también son quienes tienen una mayor proporción de victimización por actos violentos contra la persona (Stanko, 2009).

El impacto en la vida de las mujeres de su percepción de (in)seguridad es importante y las estrategias de autoprotección que desarrollan ante el miedo - ya sea a sufrir un delito, infracción administrativa o simplemente a ser "molestadas" aunque la conducta no esté recogida en ninguna normativa - limitan su libertad y suponen un obstáculo para su participación en la vida social y profesional: elegir o no una vestimenta concreta, escoger un medio de transporte $u$ otro, decidir no ir a un acontecimiento según donde se celebre o la hora de finalización, pedir que las acompañen hasta casa, rehusar un trabajo por ubicación u horarios...

Aplicar metodologías de investigación para conocer las percepciones de inseguridad y las estrategias de autoprotección o defensa que desarrollan las mujeres permitirá responder con las medidas de prevención adecuadas. A pesar de que a menudo se afirma que la percepción de (in)seguridad no se puede medir, la información sobre el sentimiento de seguridad de la población, puede objetivarse de manera clara en muchos casos: cuando la ciudadanía afirma (o se constata externamente) que hay espacios y horarios en que no lleva a cabo ciertas actividades a causa de su percepción de inseguridad en ellos, la situación se objetiva sustancialmente (Guillen, 2020). Por lo tanto, uno de los elementos claves que han de caracterizar las políticas de seguridad con perspectiva de género es admitir la importancia del componente subjetivo de la seguridad objetivable tal y como hemos manifestado- e identificar las diferentes vivencias según el género. Igualmente hay que tener en cuenta otras diferencias como la edad, la discapacidad, o la condición migratoria (Naredo, 2010).

En definitiva, la incorporación de la perspectiva de género en la seguridad también requiere tomar como punto de partida la complejidad del fenómeno, de forma que se integren: las diferentes fuentes de inseguridad, tanto las que se producen a la esfera pública como la privada; las relaciones de opresión no delictivas pero que potencian el sentimiento de inseguridad y refuerzan las estrategias de autoprotección de las mujeres; y el elemento subjetivo que genera sentimientos de inseguridad diferenciados en mujeres y hombres (Naredo, 2010).

\section{ESPACIOS SEGUROS CON PERSPECTIVA DE GÉNERO}

En relación con los dos apartados anteriores es preciso tratar de forma específica la seguridad de las mujeres en el espacio público ya que esta es una premisa indiscutible para conseguir la igualdad. Históricamente el diseño de las ciudades ha respondido a una visión androcéntrica de la vida urbana, por ello la perspectiva de género se ha de incluir dentro del urbanismo y planeamiento de las ciudades con el objetivo de eliminar las desigualdades que restan movilidad, seguridad y, por tanto, libertad a las mujeres, en el espacio público. 
Deviene imprescindible tener en cuenta los principios elaborados a partir de las experiencias de las mujeres de Montreal, que fueron recogidos con la coordinación de Anne Michaud en el documento Pour un environment urbain sécuritaire. Guide d'aménagement. Programme Femmes et Ville de la Ville de Montreal, 2002 ${ }^{14}$. Los seis principios de Montreal deben ser analizados e implantados de una manera conjunta para conseguir un espacio público seguro:

1. Señalización: Saber dónde se está y adónde se va.

2. Visibilidad: Ver y ser vista.

3. Concurrencia de personas: Oír y ser oída.

4. Vigilancia formal y acceso a la ayuda: Poder escapar y obtener auxilio.

5. Planificación y el mantenimiento de los lugares: Vivir en un ambiente limpio y acogedor.

6. Participación de la comunidad en las políticas de seguridad: Actuar en conjunto.

Las medidas que se adopten han de ser rigurosas y eficaces ${ }^{15}$, basadas en evidencias, porque ya lo decía Janes Jacobs en 1961 que "Los horribles crímenes públicos pueden ocurrir, y ocurren, en estaciones de metro bien iluminadas

$14 \quad$ En este ámbito es imprescindible citar nuevamente el trabajo realizado por el Col-lectiu Punt 6, que puede consultarse en su web http://www.punt6.org/ca/inici/

15 El asesinato de Sarah Everard, ha puesto sobre la mesa el cuestionamiento de la efectividad de algunas medidas de seguridad propuestas desde los poderes públicos basadas simplemente en aumento del alumbrado, mayor vigilancia y mayor presencia policial https://qz.com/1985650/sarah-everard-design-strategies-to-make-streets-safer-for-women/ cuando no hay ojos eficaces. Prácticamente nunca ocurren en teatros a oscuras donde hay muchas personas y ojos presentes". Por ello, ante decisiones populares pero poco eficaces como alumbrar sin medida ni criterio una $z^{z o n a}{ }^{16}$, Ilenarla de cámaras de videovigilancia o de presencia policial, cabe preguntarse si la solución no pasará por otras opciones como fomentar la solidaridad y los lazos comunitarios y dotar a los espacios de vida con actividades, equipamientos, mobiliario urbano adecuado, etc. que lo conviertan en un sitio que sea agradable de transitar y permanecer, aumentando así la presencia de personas que serán las que puedan prestar auxilio inmediato en caso de emergencia y harán disminuir la percepción de inseguridad.

En este punto aplicar el enfoque CPTED ${ }^{17}$ de prevención del delito a través del diseño ambiental con perspectiva de género puede ser de gran utilidad para aplicar estrategias que den resultados efectivos ${ }^{18}$. La perspectiva del CPTED corresponde a un abordaje multidisciplinario que busca "reducir las oportunidades de cometer delitos de oportunidad así como

16 Vid. http://agrupacionio.com/en-un-espacio-solitario-nadie-puede-oir-tus-gritos/

17 Vid. la página web de la International Crime Prevention Through Environmental Design Association donde hay materiales para profundizar sobre el enfoque CPTED https://www. cpted.net/

18 Actualmente el enfoque CPTED incluye principios de primera, segunda y tercera generación que van mucho más allá de medidas meramente físicas. Estos principios son: control de accesos, vigilancia natural, refuerzo territorial, participación comunitaria, sostenibilidad y salud pública y auto realización. 
reducir el temor de la comunidad aumentando la cohesión comunitaria" (Rau, 2005: 87)

Incluyo en este apartado el necesario estudio de la movilidad por géneros, ya que el transporte seguro ha de tener en cuenta $y$ cubrir las necesidades de las mujeres, debe fundamentarse en el reconocimiento de sus distintas experiencias, necesidades y roles distintivos.

Comparto a modo de ejemplo, algunas de las medidas que detalla el Plan de prevención del acoso sexual en las redes de metro y autobús de Transports Metropolitans de Barcelona (TMB) ${ }^{19}$ que fue presentado en mayo de 2021:

- Incrementar la presencia de vigilantes de seguridad en el metro y en el autobús (en el metro ya se aumentó un $3 \%$ en 2020 y se preveía otro aumento de $3 \%$ en 2021; en 2020 se inició también la medida de incorporar en los autobuses la vigilancia por parte de los agentes de manera selectiva).

- Implantar un sistema de video vigilancia específico dentro de los autobuses y de los metros (medida ya en desarrollo)

- Establecer un sistema de paradas a demanda en rutas de autobuses de áreas periféricas.

- Asegurar la visibilidad en las marquesinas de las paradas de autobús gestionadas por los distintos ayuntamientos implicados.

- Contar con una mayor presencia de agentes de Mossos d'Esquadra y policías locales en las redes de autobús y metro.

19 Vid. https://noticies.tmb.cat/sala-de-premsa/tmb-completa-pla-de-prevencio-de-lassetjament-sexual-xarxes-de-metro-bus
- Mejorar la iluminación de vestíbulos, andenes y pasillos.

- Estudiar la posibilidad de incorporar un hilo musical en toda la red de metro que ayude a generar un ambiente más amable y acogedor.

- El plan también incluye un protocolo de actuación de los diferentes responsables, áreas y departamentos internos de la organización ante un incidente de carácter sexual o de género que pueda producirse en las redes de metro y bus.

Encuantoalaviolenciaen espacioscomunitarios, conviene hacer una especial mención a la que se sufre por las mujeres en entornos de ocio y deportivos. En los últimos años se han adoptado por diferentes asociaciones ${ }^{20}$ y organizaciones públicas ${ }^{21}$ y privadas ${ }^{22}$ protocolos de actuación para la prevención, detección y atención a las personas afectadas por violencias sexuales. Estos protocolos han de ir necesariamente

20 La Asociación de Mujeres en el Deporte Profesional ha realizado una gran labor de sensibilización al respecto y diversas formaciones para implantar estos protocolos en entidades deportivas https://www.mujereseneldeporte.com/2018/11/ la-violencia-sexual-en-el-ambito-deportivo-prevencion-deteccion-y-atencion-voice/

21 Especialmente las administraciones locales han creado Protocolos de actuación. Véase por ejemplo el "No Callamos" del Ayuntamiento de Barcelona https://ajuntament. barcelona.cat/dones/es/protocolo-no-callamos-contra-las-agresiones-y-los-acosos-sexuales-en-espacios-de-ocio-nocturno 22 Por ejemplo, en los eventos de Live Nation con el lema \#respétame se han implantado protocolos anti acoso. Enlazo una noticia relacionada con el Download Festival Madrid de 2018 https://metaljournal.net/download-festival-madrid-implanta-un-protocolo-contra-el-acoso-sexual/ 
acompañados de una formación sobre violencias machistas a todos los miembros de la organización, una implementación de las medidas, procedimientos para la gestión de incidencias y denuncias, así como la instalación de puntos estáticos dónde se puedan dirigir las personas afectadas ${ }^{23}$. En la parte más operativa del Protocolo debe hacerse un esquema sobre cómo se va a actuar en el caso de que alguien vea o alguien denuncie una conducta que constituya violencia sexual. Es recomendable hacer una clasificación de los tipos de conductas que nos podemos encontrar y la intervención que se llevará a cabo ante cada una de ellas y por parte de quién. Para ello debe tenerse clara la legislación sobre ante qué conductas se puede intervenir y quién tiene competencia para hacerlo (Almécija, 2019).

\section{CRIMINALIDAD EN FEMENINO}

La criminología feminista - basada en evidencias, tal y como el resto de la ciencia criminológica- no niega el comportamiento delictivo femenino. La mujer ha delinquido en todos los periodos históricos, aunque en mucha menor medida que en la conducta ilícita masculina ${ }^{24}$. No contamos con datos precisos sobre la criminalidad de las mujeres ya que históricamente se realizaba una valoración de los datos de la delincuencia de forma conjunta, sin segregación por sexos, y como indica De

23 Vid. https://cuadernosdeseguridad.com/2020/04/protocolos-violencia-sexual-eventos/

24 Pueden consultarse los datos de los últimos años en los diferentes informes del Ministerio del Interior http://www. interior.gob.es/ca/web/archivos-y-documentacion/documentacion-y-publicaciones/anuarios-y-estadisticas la Cuesta (1992) esta es una medida, como se sabe, poco acertada desde el punto de vista epistemológico, porque las motivaciones para delinquir entre hombres y mujeres difieren, aunque haya algún acercamiento desde el ámbito social. Otras veces estas cifras componían un anexo de las estadísticas oficiales de la delincuencia total. Es por ello que la investigación es imprescindible para ahondar en perfiles y motivaciones que permitan avanzar en la prevención y el abordaje del delito y otras conductas ilícitas, también cuando su autora es una mujer.

Tal y como expresan Trespaderne y Garriga (2018) en su trabajo sobre los perfiles y signos de radicalización de las mujeres radicalizadas por organizaciones terroristas de etiología yihadista en España, la captación y radicalización violenta femenina no es una réplica a la masculina, y para ello deben trabajarse también, programas de prevención y desradicalización centrados en sus circunstancias particulares (Saltman y Smith, 2015).

Citando a Ennaji (2016), manifiestan que para contrarrestar las narrativas terroristas debe comprenderse antes los motivos de la radicalización violenta tanto en hombres como en mujeres.

También Caballero (2018) en una de sus investigaciones sobre Grupos Urbanos Violentos y delitos de odio trata en diversos apartados del rol de la mujer en esos grupos (representativa, nula, ornamental, cosificada, etc.) incluyendo imágenes de simbología y vestimenta para identificarlas. 
En relación con las mujeres homicidas y asesinas, Velasco (2018) afirma que ellas tienen sus propios medios y tienen sus propios motivos para acabar con la vida de sus maridos, amantes, amigos e incluso hijos.

Finalmente, hay algunas cuestiones que a pesar de ubicarse más en el ámbito de justicia que de la seguridad, deben tratarse incuestionablemente, como el contenido y fallo de las sentencias que condenan a mujeres, la influencia del género en la toma de decisiones judiciales como los tipos delictivos y los perfiles de las mujeres internas en centros penitenciarios. De ahí se puede extraer mucho conocimiento y experiencias necesarias para avanzar en el abordaje de la seguridad con perspectiva de género (incluso en el ámbito de las cárceles, investigar sobre cómo trabajar la propia seguridad de las mujeres internas).

De acuerdo con Páez-Mérida (2021) existe cierto consenso en la afirmación de que el género influye en las decisiones de los jueces, no queda tan claro de qué manera lo hace. Tal y como afirma la autora citada, aunque los estudios sobre sentencing y género cuenten con limitaciones metodológicas, es posible que la disparidad de género de decisiones judiciales sea producto de las diferencias entre la delincuencia masculina y la femenina (Pina-Sánchez \& Harris, 2020): las mujeres cometen un porcentaje muy pequeño de delitos en comparación con los hombres y, además, sus delitos son menos graves (Heidensohn, 1994), lo cual justificaría la imposición de penas más leves. Estas cuestiones podrían justificar, legítimamente, que las mujeres reciban un trato más benévolo. Páez-Mérida apunta que sin embargo, no todas las mujeres son beneficiarias de ese "trato benévolo": las chicas del sistema de justicia juvenil son objeto de respuestas judiciales más severas que los chicos: se les impone un mayor número de medidas de libertad vigilada e internamiento, reciben una supervisión más intensiva que los chicos y, en definitiva, se ven más afectadas por el paternalismo judicial (Cea d'Ancona, 1992; Spivak et al., 2014). En el mismo sentido, las mujeres pertenecientes a minorías, procedentes de clases sociales bajas y que no cumplen con el estereotipo tradicional de feminidad tampoco se benefician de un trato benévolo (Kruttschnitt, 1980).

En cuanto a las mujeres internas en centros penitenciarios afirma Yugueros (2013) que las causas por las cuales las mujeres delinquen son debidas mayoritariamente a la pobreza, exclusión social, falta de instrucción educativa y otras circunstancias socioculturales y que este es precisamente el perfil que presenta la mayoría de las mujeres que cumplen condena en las cárceles españolas.

\section{MUJERES ESPECIALMENTE VULNERABLES}

El conocimiento sobre las necesidades específicas de las personas con discapacidades es imprescindible para planificar unas correctas políticas de seguridad que las protejan adecuadamente. También es una capacitación con la que deben contar las personas que han de interaccionar con ellas, por ejemplo, cualquier profesional del circuito de atención a víctimas o el personal de seguridad que hace una labor más operativa.

Pondré como ejemplo la interacción con las mujeres con autismo por ser una discapacidad 
no visible que no ha sido todavía lo suficientemente investigada con perspectiva de género ${ }^{25}$. A pesar de que en España se han publicado algunos manuales dirigidos a organizaciones policiales ${ }^{26}$, aún se pueden leer en ellos algunas afirmaciones que repiten prejuicios ("Tener en cuenta esta ausencia de empatía al valorar el testimonio" ${ }^{27}$ y en ninguno de ellos existe una perspectiva de género que señale las entre las diferencias entre un hombre con autismo y una mujer.

En cuanto a la formación policial, en España hay organizaciones policiales que han recibido formación sobre este ámbito, pero no constan datos de cuántas ni de qué tipo de formación (duración, contenidos, etc...). Al respecto, y trasladándonos a Estados Unidos, destaco el artículo de Elissa Ball y Jaclyn Jeffrey-Wilensky (2020) "Why autism training for police isn't

25 Vid. https://www.publico.es/sociedad/mujeres-descubren-40-anos-autismo-evaluaciones-rigen-criterios-machistas. html

26 En la página del Ministerio de Interior podemos encontrar la "Guía de Intervención Policial con Personas con Discapacidad Intelectual" (Vid. http://www.interior.gob.es/documents/642317/1201295/Guialntervenci\%C3\%B3nPolicialPDlversi\%C3\%B3nweb.pdf/806b2414-8c6b-483a-a928-434daf3d5dc3) También se ha publicado el "Manual de procedimiento para la atención de la Policia Local a las personas con discapacidad intelectual" editado por Plena inclusión y Unijepol, con la colaboración de la Plataforma por la Gestión Policial de la Diversidad ( $\mathrm{v}$.

https://www.plenainclusion.org/publicaciones/buscador/ manual-de-procedimiento-para-la-atencion-de-la-policia-local-a-las-personas-con-discapacidad-intelectual/)

27 Puede encontrarse esa frase en la misma guia a la que se hace referencia en la nota 11 enough" ${ }^{28}$ donde se critica la formación policial sobre el autismo señalándose que muchos departamentos de policía ofrecen capacitación en autismo, pero las sesiones suelen ser opcionales y varían enormemente en duración, formato y calidad, además pueden realizarse capacitaciones más o menos extensas sobre el tema pero hay una carencia en cuanto a evaluar si cuando se ha llevado a la práctica lo aprendido se ha realizado un actuación exitosa o no. Asimismo, las autoras destacan que habría que revisar los contenidos para saber si se está enseñando la parte "más catastrófica del autismo" o también se mencionan las fortalezas y virtudes de los autistas, porque entonces las formaciones lo que provocan es estigmatizar aún más y alimentar los prejuicios.

Asimismo, la protección e interacción con colectivos vulnerables como las niñas, mujeres mayores, inmigrantes, sin hogar, prostitutas, con dependencias, son algunos de los ámbitos que deben desarrollarse no solo desde la asistencia social sino de su seguridad: qué medidas son realmente efectivas para proteger a estas mujeres analizando los riesgos y amenazas específicas que sufren como colectivo, pero también individualmente. (también es un error considerar que todas las mujeres del mismo colectivo tienen las mismas necesidades).

\section{LAS PROFESIONALES DE LA SEGURIDAD}

En España, las mujeres representan entre un 8\% y un $21 \%$ en la seguridad pública dependiendo

\footnotetext{
28 Vid. https://www.spectrumnews.org/news/why-autism-training-for-police-isnt-enough/
} 
de la fuerza y cuerpo de seguridad al que se haga referencia y un $13 \%$ en seguridad privada ${ }^{29}$. El primer reto, pero no el único, es aumentar esa representatividad, y aunque podría parecer, por tanto, que con conseguir a medio o largo plazo la paridad en las organizaciones - imprescindible también en puestos de responsabilidad- sería suficiente, no es así ya que una mujer que trabaja en la seguridad pública o privada no tiene por qué estar trabajando desde un enfoque feminista con perspectiva de género, sino puede estar simplemente repitiendo mensajes, roles y procedimientos aprendidos $\mathrm{u}$ ordenados por sus superiores en ausencia de esa perspectiva. Por tanto, la presencia paritaria de mujeres es imprescindible y necesaria pero no suficiente. Toda la organización - y no solo ellas- debe ser sensible, tener formación en igualdad e implantar medidas desde ese enfoque.

La Ley Orgánica estatal 3/2007, de 22 de marzo, para la igualdad efectiva de mujeres y hombres, en su artículo 67 establece que las normas reguladoras de las Fuerzas y Cuerpos de Seguridad del Estado han de promover la igualdad efectiva entre mujeres y hombres, impidiendo cualquier situación de discriminación profesional, especialmente, en el sistema de acceso, formación, ascensos, destinos y situaciones administrativas. Asimismo, las normas referidas al personal al servicio de las Administraciones públicas en materia de igualdad, prevención de la violencia de género y conciliación de la vida personal,

29 Vid. https://www.seguritecnia.es/actualidad/dia-internacional-de-la-mujer-la-presencia-de-la-mujer-en-el-sistema-de-seguridad-nacional_20210306.html familiar y profesional serán de aplicación en las Fuerzas y Cuerpos de Seguridad del Estado, adaptándose, en su caso, a las peculiaridades de las funciones que tienen encomendadas, en los términos establecidos por su normativa específica.

En cuanto a las empresas de seguridad privada, la Resolución de 18 de noviembre de 2020, de la Dirección General de Trabajo, por la que se registra y publica el Convenio colectivo estatal de las empresas de seguridad para el año $2021^{30}$, establece - como así lo hacían los anteriores- que todas las empresas sujetas al convenio deben contar con un plan de igualdad con los contenidos establecidos en la Ley, cuyo objetivo será potenciar la igualdad de trato y oportunidades en el ámbito laboral.

En las organizaciones policiales y de seguridad privada algunos de los puntos esenciales a trabajar serían los siguientes:

- Contar con un Plan de igualdad e implementarlo.

- Elaborar y llevar a cabo una estrategia de comunicación externa e interna del Plan de igualdad.

- Contar con protocolos de actuación en cuanto a la prevención, detección y abordaje de las conductas que supongan acoso sexual, discriminación sexual o por razón de sexo.

- Promocionar de la transversalidad de género en las políticas de seguridad

- Garantizar la igualdad efectiva en el

30 Vid. https://www.boe.es/diario_boe/txt.php?id=BOE-A-2020-15038 
acceso y a carrera profesional del personal, promoviendo acciones para equilibrar la presencia de ambos géneros.

- Promover la investigación: informes, estudios y estadísticas sobre la igualdad de mujeres y hombres en la organización.

- Visibilizar de la mujer profesional de la seguridad y analizar la imagen que se da de ella en la prensa, carteles, campañas, videos de sensibilización....

- Contar con las mujeres profesionales de la seguridad como formadoras, ponentes, promoviendo su participación en cursos, congresos, jornadas...

- Uso de un lenguaje inclusivo.

- Formación en igualdad y perspectiva de género.

- Prevención de riesgos laborales atendiendo a las necesidades específicas de las mujeres.

- Promover de forma específica la incorporación de mujeres con discapacidad.

En cuanto a la formación, la Ley de 2007, antes citada, regula que las Administraciones Públicas debe fomentar la formación en igualdad, tanto en el acceso al empleo público como a lo largo de la carrera profesional. Además, todas las pruebas de acceso al empleo público de la Administración General del Estado y de los organismos públicos vinculados o dependientes de ella han de contemplar el estudio y la aplicación del principio de igualdad entre mujeres y hombres en los diversos ámbitos de la función pública.

También se establece que la Administración General del Estado y los organismos públicos vinculados o dependientes de ella han de impartir cursos de formación sobre la igualdad de trato y oportunidades entre mujeres y hombres y sobre prevención de la violencia de género, que se dirigirán a todo su personal.

En cuanto a la seguridad privada, de competencia estatal, en el temario mínimo obligatorio de formación previa para vigilantes que establece el Ministerio del Interior $^{31}$ no se contempla formación en igualdad, en perspectiva de género ni en violencia sexual o violencia machista en general (solo se recoge un epígrafe sobre violencia de género - que de acuerdo con el Código Penal español se limita a la que ejerce el hombre sobre la mujer que sea o haya sido su pareja- en el ámbito de la formación en derecho penal).

\section{EXPERIENCIAS EN CATALUÑA}

En Cataluña hay que destacar diferentes experiencias, protocolos de actuación y artículos concretos de normativas que incorporan la perspectiva de género en la seguridad $^{32}$. Además, el plan de Gobierno de la IV legislatura de la Generalitat de Cataluña, aprobado el 21 de septiembre de $2021^{33}$, ha

$31 \quad$ Puede consultarse en la página web del Ministerio del Interior http://www.interior.gob.es/ca/web/servicios-al-ciudadano/personal-de-seguridad-privada/vigilantes-de-seguridad/ temario

32 Sirva como ejemplo los contenidos tratados en las dos Jornadas realizadas en 2019 y 2021 sobre planificación de la seguridad con perspectiva de género que organizó la Universidad Politécnica de Cataluña. Pueden consultarse los programas en https://segues.upc.edu/es/jornadas-femsegues

33 Vid. https://presidencia.gencat.cat/ca/ambits_d_actuacio/pla-de-govern/ 
hecho una apuesta firme por feminizar los cuerpos de policía, agentes rurales y bomberos, comprometiéndose a aprobar e implementar los planes de igualdad para impulsar políticas activas en material de igualdad de género en estos cuerpos operativos y conseguir, además, la paridad en la estructura directiva de los Mossos d'Esquadra en 2030.

\section{NORMATIVA}

En España, existe por una parte una norma estatal a la que se ha hecho referencia anteriormente, la Ley Orgánica 3/2007 para la igualdad efectiva de mujeres y hombres ${ }^{34}$, y por otro las normas de las Comunidades Autónomas que regulan esa misma materia, en virtud de sus competencias de autogobierno.

En ningún caso estas Leyes autonómicas hacen una mención específica a la incorporación de la perspectiva de género en la seguridad, con la excepción de la Ley 17/2015, de 21 de julio ${ }^{35}$, aprobada por Cataluña, que regula en su artículo 55 que el Departamento competente en materia de seguridad debe:

a) Tener en cuenta la distinta percepción de la seguridad de mujeres y hombres e incluir la perspectiva de género en las encuestas de victimización, en el análisis de la realidad de la seguridad en Cataluña, en las diagnosis de seguridad de los espacios públicos, así como en la definición de las políticas y en la adopción

\footnotetext{
34 Vid. https://www.boe.es/buscar/act.php?id=BOE-A-2007-6115

35 Vid. https://www.boe.es/diario_boe/txt.php?id=BOE-A-2015-9676
}

de medidas de prevención y protección relacionadas con la autonomía personal y el uso de los espacios, tanto públicos como privados.

b) Establecer las medidas necesarias para erradicar la violencia machista a nivel comunitario.

c) Promover el trabajo con los hombres a fin de que no ejerzan ningún tipo de violencia machista.

Asimismo, en el mismo artículo 55, se establece que el Departamento competente en materia de urbanismo debe:

a) Promover un modelo de seguridad que incorpore la perspectiva de las mujeres en el planeamiento urbanístico. Con esta finalidad el planeamiento debe vincular diseño urbano y violencia machista, para evitar entornos y elementos que puedan provocar situaciones de inseguridad para las mujeres.

b) Erradicar la percepción de inseguridad en los espacios públicos, garantizando en ellos la visibilidad, correcta iluminación y alternativa de recorridos, y dotándolos de usos y actividades diversos, intergeneracionales y que fomenten la presencia de gente diversa.

Este precepto de la legislación catalana es de gran relevancia ya que por fin se recoge en una norma con rango Ley muchas de las cuestiones que se tratan en el presente artículo, por lo tanto, lejos de ser una "buena práctica opcional", existe una obligación por parte de los poderes públicos de trabajar la seguridad con perspectiva de género. Además, no solo debe hacerse desde el departamento competente en 
seguridad, sino también desde el competente en urbanismo, con lo que se relaciona directamente con enfoques criminológicos como el CPTED, así como con los seis principios de Montreal anteriormente citados.

\section{LA FORMACIÓN}

También se regula por Ley la formación con la que se debe contar en las organizaciones policiales. La Ley catalana de 2015, establece que los temarios para el acceso al empleo público deben incluir los contenidos relativos a la normativa sobre igualdad efectiva de mujeres y hombres y sobre violencia machista que deben aplicarse en la actividad administrativa y en el desarrollo de las políticas de igualdad efectiva.

Además, la Ley 5/2008, de 24 de abril, del derecho de las mujeres a erradicar la violencia machista $^{36}$ regula que las administraciones públicas de Cataluña deben garantizar que los cuerpos policiales dispongan de la adecuada formación básica en materia de violencia machista y de la formación y capacitación específicas y permanentes en materia de prevención, asistencia y protección de las mujeres que sufren violencias.

En 2020 se creó la Comisión para la igualdad de género de la Dirección General de la Policía ${ }^{37}$, en la que se indica que una de las funciones de la comisión es velar por que todo el personal

36 Vid. https://www.boe.es/buscar/act.php?id=BOE-A-2008-9294

37 Vid. https://govern.cat/salapremsa/notes-premsa/383211/cos-mossos-esquadra-aposta-organitzacio-policial-perspectiva-genere-equitat de la Dirección tenga formación en perspectiva de género, y especialmente todo el personal de los cargos de mando. Además, se ha creado la Unidad de Igualdad y Equidad integrada por policías con formación en políticas de igualdad de género y referentes en la materia y en su gestión pública.

\section{LAS MUJERES EN EL SECTOR DE LA SEGURIDAD EN CATALUÑA}

La unidad de investigación de la Escuela de Policía del Instituto de Seguridad Pública de Cataluña presentó en 2020 un interesante trabajo sobre cómo incorporar más mujeres a la policía (Vallès, Vivolas y Egea, 2020). En el estudio se plasma como las mujeres todavía son minoría, tanto en los cuerpos policiales como en los procesos de selección y formación básica. Destaco las medidas directas e indirectas que se recogen que han demostrado su efectividad para incorporar más mujeres en la policía:

- Entre las directas se encuentran las campañas de contratación; las medidas de apoyo en la preparación de las pruebas de acceso, la adaptación de los criterios de selección y de los tribunales evaluadores y la discriminación positiva.

- Las indirectas inciden en hacer la organización más atractiva para las mujeres con medidas de apoyo a la promoción; de adaptación física de la organización; de aumento de la transparencia y reducción de la discriminación y para favorecer la conciliación familiar.

Además, cabe destacar que en el último año se han producido dos modificaciones normativas con la finalidad de promover este acceso de 
las mujeres a las policías de Cataluña, tanto en relación a la policía autonómica como a las policías locales.

En primer lugar, se ha añadido una disposición transitoria séptima a la Ley 10/1994, de 11 de julio, de la Policía de la Generalitat-Mossos $d^{\prime} E^{\prime}$ quadra ${ }^{38}$ en la que se indica que mientras no se apruebe el correspondiente plan de igualdad, y con el fin de alcanzar el equilibrio en el número de mujeres y hombres en la plantilla del cuerpo de Mossos d'Esquadra, las convocatorias para acceder a las diferentes categorías de este cuerpo policial tienen que establecer que los empates de puntuación en el orden de clasificación de los aspirantes deben dirimirse, como primer criterio, dando preferencia a las personas candidatas del sexo menos representado cuando este no llegue al 40\% de aquella categoría.

La segunda modificación ha sido a la Ley 16/1991, de 10 de julio, de las Policías Locales, donde se ha añadido una disposición adicional 8 por la Ley 5/2020 de 29 de abril, en la que se regula que las convocatorias para acceder a las diferentes categorías de policías locales y vigilantes municipales aprobadas a partir del 1 de enero de 2020, deben determinar el número de plazas que han de ser provistas por mujeres para cumplir el objetivo de equilibrar la presencia de mujeres y hombres en las plantillas respectivas.

38 La disposición transitoria séptima ha sido añadida por el artículo único del Decreto Ley 1/2021, de 12 de enero por el cual se modifica la Ley 10/1994, de 11 de julio de la Policía de la Generalitat-Mossos d'Esquadra y se establecen medidas correctoras para equilibrar la presencia de mujeres y hombres.
El número de plazas reservado para mujeres debe ser proporcional a los objetivos perseguidos, y no puede ser superior al $40 \%$ de las plazas convocadas ni inferior al porcentaje que sea establecido por el plan de igualdad de cada ayuntamiento. Como regla general, hasta que los ayuntamientos no elaboren estos planes de igualdad, el porcentaje mínimo no puede ser inferior al $25 \%$ de las plazas convocadas, siempre que se convoquen más de tres.

En estos procedimientos selectivos, la adjudicación de las plazas convocadas debe realizarse siguiendo una única lista final de las personas que hayan superado el proceso, ordenadas según la puntuación obtenida, aplicando los criterios de desempate establecidos legalmente, salvo que por este procedimiento no se alcance el porcentaje del 25-40\%, en cuyo caso se deberá dar preferencia a las candidatas mujeres, hasta alcanzar el objetivo perseguido, siempre que se cumplan las siguientes condiciones:

a) Tiene que haber en todos los casos una equivalencia de capacitación, determinada por la superación de las pruebas y los ejercicios de la fase de oposición del sistema selectivo.

b) Ninguna de las candidatas mujeres seleccionadas en virtud de este criterio de preferencia puede tener un diferencial negativo de puntuación, en la fase de oposición y, si procede, en la fase de concurso, de más del $15 \%$ respecto a los candidatos hombres preteridos.

c) No puede aplicarse esta medida respecto a candidatos en los que concurran motivos de discriminación positiva legalmente determinados otros que el criterio de 
preferencia que regula la presente disposición, como la pertenencia a otros colectivos con especiales dificultades de acceso al empleo.

El criterio de preferencia que regula la disposición no será aplicable si en el cuerpo, la escala y la categoría objeto de la convocatoria hay una presencia de funcionarias mujeres igual o superior al $33 \%$.

\section{ALGUNAS EXPERIENCIAS EN CATALUÑA}

\section{Ayuntamiento de Barcelona. Bases para la convocatoria de plazas de la Guardia Urbana}

Para promover la igualdad entre las personas aspirantes a una plaza de agente Guardia Urbana, el Ayuntamiento de Barcelona, en las bases de las convocatorias prevé que si en el día y hora de la realización de las pruebas del proceso selectivo - en relación a las pruebas culturales, teóricas, aptitudinales, de inglés o conocimiento de la lengua catalana - alguna de las mujeres aspirantes está hospitalizada por embarazo de riesgo, parto o los días inmediatamente posteriores al parto, se debe articular por el Tribunal los mecanismos necesarios para realizar la prueba correspondiente en el recinto hospitalario donde se encuentre, siempre que sea dentro de la provincia de Barcelona y la persona lo solicite en la forma y plazo que establece la convocatoria. Igualmente, en el caso que alguna de las mujeres aspirantes sea madre lactante y considere que la realización de las pruebas coincidirá con la lactancia de su bebé lo ha de notificar.
En relación a las pruebas de aptitud físicas, las mujeres aspirantes que el día de la prueba estén embarazadas o que no hayan cumplido las 16 semanas desde el nacimiento de su hijo o hija pueden realizar la prueba - aportando un certificado médico donde expresamente se indique que no hay ningún peligro para su realización - o pedir un aplazamiento hasta la siguiente convocatoria, manteniendo la nota de las pruebas que ya hagan realizado siempre que en el siguiente proceso de selección se mantengan los mismos baremos y condiciones.

Además, de acuerdo con lo establecido por la disposición adicional 8 a de la Ley 16/1991, antes citada, en la última convocatoria se reservaron el 40 por ciento de las plazas para mujeres.

Finalmente, en caso de empate en la última posición, se establece como criterio de desempate que se favorecerá a la persona aspirante del género que sea menos representado en el colectivo profesional.

\section{El Protocolo de seguridad contra las violencias sexuales en entornos de ocio}

En 2019 el Departamento de Interior aprobó el Protocolo de seguridad contra las violencias sexuales en entornos de ocio ${ }^{39}$, al cual se pueden adherir entidades públicas y privadas organizadoras de eventos como fiestas mayores, salas de fiestas, festivales de música, etc. Esta adhesión conlleva unos compromisos de formación de todo el personal (operativo y no

39 Vid. https://dogc.gencat.cat/es/document-del-dogc/ index.html?documentld=846635 
operativo) en violencias machistas desde el punto de vista de la sensibilización, su conocimiento desde el punto de vista normativo y también cómo articular procedimientos para su prevención, detección y actuación en caso de producirse, así como de atención a las personas afectadas. Durante el transcurso del evento, además, deben implantarse medidas para la prevención y abordaje de las violencias sexuales, entre ellas, instalar un punto de atención e información de las violencias sexuales.

Entre las medidas, se prevén algunas que están relacionadas con el enfoque que propone la prevención situacional. Se establece que los titulares, organizadores o promotores de las actividades de ocio tienen que analizar los diferentes espacios de sus instalaciones, así como los espacios públicos que conforman el entorno de la actividad, para identificar posibles zonas de riesgo que puedan propiciar la comisión de violencias sexuales, ya sea porque se trata de zonas apartadas, con poca visibilidad, con condiciones de falta de iluminación, o que dificultan poder pedir ayuda en caso de sufrir algún hecho, etc. En la medida en que sea posible, se tendrán que adoptar medidas compensatorias de los riesgos detectados, ya sea reforzando la iluminación, haciendo verificaciones en estas zonas, instalando mecanismos de alarma, etc., en función de las características de los lugares y de los riesgos detectados.

\section{El Protocolo para el abordaje de las infracciones de odio y discriminación para las policías locales}

También en 2019 se aprobó por el Departament d'Interior, el Protocolo para el abordaje de las infracciones de odio y discriminación para las policías locales de Cataluña ${ }^{40}$, que está destinado a dotar a las policías locales de pautas de actuación operativas para la prevención, detección y gestión de situaciones relacionadas con el odio y la discriminación, la asistencia a las víctimas, concretar actuaciones con las personas presuntamente autoras e identificar especialidades en la confección de la documentación policial para facilitar la tramitación de las denuncias por las autoridades judiciales y administrativas. En cuanto al ámbito que nos ocupa en este artículo, es de destacar que también define y establece ta de cómo abordar los actos de odio o de discriminación por motivos de sexo y por razón de sexo (además de otros como la orientación sexual e identidad de género, motivos religiosos, enfermedad, raza, etnia, nación, etc...)

\section{LA PERCEPCIÓN IMPORTA}

En 2021, se presentó por el Departament d'Interior de la Generalitat de Cataluña, en el marco del proyecto europeo Cutting Crime Impact $(\mathrm{CCl})^{41}$, la herramienta "La percepció importa. La seguretat de les persones" 42 elaborada en colaboración con técnicos municipales (básicamente del Ayuntamiento de Barcelona) y altos mandos de organizaciones policiales (Mossos d'Esquadra y policías

$40 \quad$ Vid. https://dogc.gencat.cat/es/document-del-dogc/?documentld $=857274$

$41 \quad$ Vid. https://www.cuttingcrimeimpact.eu/

42 Puede descargarse en su versión en castellano el siguiente enlace https://interior.gencat.cat/web/.content/ home/010_el_departament/publicacions/seguretat/Toolkit/ booklet_Digital_ES_no_20210611.pdf 
locales). Se trata de una herramienta práctica para analizar manifestaciones de percepción de inseguridad y articular respuestas eficaces. Los gestores públicos de la seguridad se encuentran en situaciones en que las expresiones públicas de inseguridad requieren una interpretación y toma de decisiones que permita gestionar la situación de manera que no se deteriore. La herramienta contiene un capítulo dedicado a la perspectiva de género, $y$, además, este enfoque se aplica de forma transversal en todo su contenido.

\section{TRABAJOS CITADOS}

Almécija, A. (2019) La prevención de la violencia sexual en espacios de ocio: iniciativas desde la administración local. La Administración Práctica, № 9, págs-45-52

Ayguasenosa i Soro, N. (2009) La gestió alternativa de conflictes (GAC) aplicada a la seguretat. Revista Catalana de Seguretat Pública, Núm. 21, p. 121-38, https://raco.cat/index.php/ RCSP/article/view/166851

Burgos, A. (Coord) 5º Informe anual 2017-2018. Observatorio Noctámbul@s (2018) https://www.drogasgenero.info/wpcontent/uploads/5InformeNoctambulas_2017-18.pdf

Caballero, J. (2018) Los Grupos Urbanos Violentos y los delitos de odio. El uso de la simbología violenta como determinante al comportamiento criminal. Herramientas para los profesionales para detectar el discurso y los símbolos de odio de los Grupos Urbanos Violentos. Centro de Estudios Jurídicos y Formación Especializada. Generalitat de Catalunya. http://cejfe.gencat.cat/es/recerca/cataleg/ crono/2018/grups-urbans-violents/

Campbell, R., Adams, A., Wasco, S., Courtney, A. y Self, T. (2009). Training interviewers for research on sexual violence: a qualitative study of rape survivors' recommendations for interview practice. Violence Against Women, 15(5): 595-617 En
Cea d'Ancona, M.A. (1992). La justicia de menores en España. Centro de Investigaciones Sociológicas. En Páez-Mérida, A. (2021). Estado de la cuestión del estudio de la influencia del género en la toma de decisiones judiciales. Revista Española De Investigación Criminológica, 19(1). https://doi. org/10.46381/reic.v19i1.483

Guillén, F. (2020). La falacia de la seguridad objetiva y sus consecuencias. A International e-Journal of Criminal Sciences. Article 4, Num. 15 (2020)

Ennaji, M. (2016). Recruitment of foreign male and female fighters to Jihad: Morocco's multifaceted counter terror strategy. International Review of Sociology, 26 (3), 546 - 557. En Trespaderne y Garriga (2018) Perfiles y signos de radicalización de las mujeres radicalizadas por organizaciones terroristas de etiología yihadista en España. Behavior \& Law Journal, ISSN-e 2444-4170, Vol. 4, №. 1, págs. 21-28 https://dialnet.unirioja.es/servlet/ articulo?codigo $=7742028$

Freixanet, M. (coord.) No surtis sola: espais públics segurs amb perspectiva de gènere: ciutats $\mathrm{i}$ persones. Institut de Ciències Polítiques i Socials (2011).

García Sánchez, A. (2009) Cap a un model estandarditzat d'atenció policial a les dones que pateixen violència masclista. Revista Catalana de Seguretat Pública, Núm. 20, p. 13-26, https://raco.cat/index.php/RCSP/article/ view/143076

Heidensohn, F. (1994). Gender and Crime. En M. Maguire, R. Morgan \& R. Reiner. (Eds.). (1994). The Oxford Handbook of Criminology(pp. 997-1039). Clarendon Press. En PáezMérida, A. (2021). Estado de la cuestión del estudio de la influencia del género en la toma de decisiones judiciales. Revista Española De Investigación Criminológica, 19(1). https://doi.org/10.46381/reic.v19i1.483

Igareda, N., \& Bodelón, E. (2014). Las violencias sexuales en las universidades: cuando lo que no se denuncia no existe. Revista Española De Investigación Criminológica, 12, 1-27. https://doi.org/10.46381/reic.v12i0.79

Jordan, J. (2001). Worlds apart? Women, rape and the reporting process. British Journal of Criminology 41 (4): 679-706. En 
Igareda, N., \& Bodelón, E. (2014). Las violencias sexuales en las universidades: cuando lo que no se denuncia no existe. Revista Española De Investigación Criminológica, 12, 1-27. https://doi.org/10.46381/reic.v12i0.79

Jordan, J. (2004). The Word of a Woman? Police, Rape and Belief. Houndmills, Basingstoke: Palgrave Macmillan. En Igareda, N., \& Bodelón, E. (2014). Las violencias sexuales en las universidades: cuando lo que no se denuncia no existe. Revista Española De Investigación Criminológica, 12, 1-27. https://doi.org/10.46381/reic.v12i0.79

Jordan, J. (2008). Serial Survivors: Women's Narratives of Surviving Rape. Sydney: The Federation Press. En Igareda, N., \& Bodelón, E. (2014). Las violencias sexuales en las universidades: cuando lo que no se denuncia no existe. Revista Española De Investigación Criminológica, 12, 1-27. https://doi.org/10.46381/reic.v12i0.79

Jordan, J. (2012). Silencing rape, silencing women. En Brown, J. M. y Waklate, S. L. Handbook on sexual Violence, London: Routledge En Igareda, N., \& Bodelón, E. (2014). Las violencias sexuales en las universidades: cuando lo que no se denuncia no existe. Revista Española De Investigación Criminológica, 12, 1-27. https://doi.org/10.46381/reic.v12i0.79

Kruttschnitt, C. (1980). Socialstatus and sentences of female offenders. Law \& Society Review, 15(2), 247 266. https://doi.org/10.2307/3053604 En Páez-Mérida, A. (2021). Estado de la cuestión del estudio de la influencia del género en la toma de decisiones judiciales. Revista Española De Investigación Criminológica, 19(1). https://doi. org/10.46381/reic.v19i1.483

Michaud, A. (coord.). Guide d'amenagement pour un environnement urbain sècuritaire. Femmes et ville. Ville de Montreal, 2003.

Michaud, A. (2005): Seguridad y convivencia: un flujo con enfoque de género en el espacio urbano En Velázquez, I. (coord.), Urbanismo y género. Una visión necesaria para todos. Barcelona: Diputación de Barcelona.

Naredo, M. (2010). Guia per a l'elaboració de diagnòstics de gènere a l'àmbit rural i urbà. Departament d'Interior de la Generalitat de Catalunya.
Naredo, M. (2011): «Metodologías para la detección de problemas de seguridad con enfoque de género». En M. FREIXANET (coord.), «No surtis sola». Espais públics segurs amb perspectiva de gènere, Barcelona: Institut de Ciències Polítiques i Socials, Universitat Autònoma de Barcelona.

Ortiz, S. (2014) Espacio público, género e (in)seguridad. En Cortés, C. (coord.) Jornadas Urbanismo y Género. Ciudades en Construcción. Perséfone. Ediciones electrónicas de la AEHM/UMA. (p. 48-67)

Páez-Mérida, A. (2021). Estado de la cuestión del estudio de la influencia del género en la toma de decisiones judiciales. Revista Española De Investigación Criminológica, 19(1). https://doi.org/10.46381/reic.v19i1.483

Pina-Sánchez, J. \& Harris, L. (2020). Sentencing Gender? Investigating the Presence ofGender Disparities in Crown Court Sentences. Criminal Law Review, 1(1), 3-28 En PáezMérida, A. (2021). Estado de la cuestión del estudio de la influencia del género en la toma de decisiones judiciales. Revista Española De Investigación Criminológica, 19(1). https://doi.org/10.46381/reic.v19i1.483

Quivy, R. y Van Campenhoundt, L. (1992). Manual de investigación en ciencias sociales. Mexico, D.F.: Limusa Noriega En Igareda, N., \& Bodelón, E. (2014). Las violencias sexuales en las universidades: cuando lo que no se denuncia no existe. Revista Española De Investigación Criminológica, 12, 1-27. https://doi.org/10.46381/reic.v12i0.79

Rau, M. (2005). Prevención del crimen mediante el diseño ambiental en Latinoamérica. Un Ilamado a la acción ambiental comunitaria. Ciudad y seguridad en américa latina

Shreeves, R. (2019) La integración de la perspectiva de género en la UE: situación actual - Servicio de Estudios del Parlamento Europeo. Servicio de Estudios para los Diputados.

Spivak, A. L., Wagner, B. M., Whitmer, J. M.\& Charish, C. L. (2014). Gender and Status Offending: Judicial Paternalism in Juvenile Justice Processing. Feminist Criminology, 9(3), 224-248. https://doi.org/10.1177/1557085114531318 En Páez-Mérida, A. (2021). Estado de la cuestión del estudio de la influencia del género en la toma de decisiones judiciales. Revista Española De Investigación Criminológica, 19(1). 
https://doi.org/10.46381/reic.v19i1.483

Stanko, E. (1990). Everyday violence. How women and men experience sexual and physical danger. London: Pandora En Igareda, N., \& Bodelón, E. (2014). Las violencias sexuales en las universidades: cuando lo que no se denuncia no existe. Revista Española De Investigación Criminológica, 12, 1-27. https://doi.org/10.46381/reic.v12i0.79

Stanko, E. (2009) És pot reduir la por de la delinqüència que tenen les dones?. Revista Catalana de Seguretat Pública, 2009, Núm. 20, p. 47-60, https://raco.cat/index.php/RCSP/ article/view/143078

Trespaderne y Garriga (2018) Perfiles y signos de radicalización de las mujeres radicalizadas por organizaciones terroristas de etiología yihadista en España. Behavior \& Law Journal, ISSN-e 2444-4170, Vol. 4, №. 1, págs. 21-28 https://dialnet. unirioja.es/servlet/articulo?codigo $=7742028$

Vallés, M. S. (1997). Técnicas cualitativas de investigación social. Reflexión metodológica y práctica profesional. Madrid: Síntesis. En Igareda, N., \& Bodelón, E. (2014). Las violencias sexuales en las universidades: cuando lo que no se denuncia no existe. Revista Española De Investigación Criminológica, 12, 1-27. https://doi.org/10.46381/reic. v12i0.79

Vallés, M. (2002). Entrevistas cualitativas. Madrid: Centro de Investigaciones Sociológicas. En Igareda, N., \& Bodelón, E. (2014). Las violencias sexuales en las universidades: cuando lo que no se denuncia no existe. Revista Española De Investigación Criminológica, 12, 1-27. https://doi.org/10.46381/reic.v12i0.79

Vallès, L, Vivolas, B. Egea, A. (2020) Com incorporar més dones a la policia. Estat de la qüestió i propostes de futur. Unitat de Recerca de I'Escola de Policia de I'Institut de Seguretat Pública de Catalunya

Velázquez, I. (coord.), Urbanismo y género. Una visión necesaria para todos. Barcelona: Diputación de Barcelona. 2006

Yugueros, A.J. (2013) La delincuencia femenina: una revisión teórica Foro, Nueva época, vol. 16, núm. 2: 311-316

\section{Ana Belén Almécija Casanova}

Afiliación: Escuela Politécnica Superior de Edificación de Barcelona de la Universidad Politécnica de Cataluña

Licenciada en Derecho y en Criminología. Profesora asociada en la Escuela Politécnica Superior de Edificación de Barcelona de la Universidad Politécnica de Cataluña. Experta profesional en dirección y gestión de la seguridad integral. Técnica en la elaboración de planes de autoprotección. Técnica en gestión de riesgos y protección civil. Agente y promotora de igualdad. Actualmente trabaja en el Departamento de Interior de la Generalitat de Cataluña. Correo de contacto annaalmecijasp@gmail.com 